Iuri ZAPPI

\title{
Prima segnalazione per l'Italia di Tarsostenus carus (Coleoptera Cleridae)
}

\begin{abstract}
Riassunto: Viene segnalata per la prima volta la presenza in Italia di Tarsostenus carus (Newman, 1840) sulla base di un esemplare femmina raccolto nelle Marche nel 1999. La specie è originaria della regione australiana e risulta essersi già acclimatata in Europa, in particolare in Gran Bretagna e Francia. Vengono fornite brevi notizie sulla sua tassonomia, biogeografia ed ecologia.
\end{abstract}

\begin{abstract}
First record of Tarsostenus carus (Newman, 1840) for Italy (Insecta, Coleoptera, Cleridae).
Tarsostenus carus (Newman, 1840) is reported for the first time from Italy, from a female specimen sampled in 1999 in central Italy (Osimo, Ancona, Marche). This beetle is native from the Australian region and results already acclimatized in Europe, in particular in Great Britain and France. Information on taxonomy, biogeography and ecology of the species is given.
\end{abstract}

Key words: Cleridae, Tarsosteninae, Tarsostenus carus, New species to Italy, Marche.

\section{INTRODUZIONE}

Tarsostenus carus (Newman, 1840) è un piccolo coleottero Cleridae originario della regione australiana (Australia, Nuova Caledonia, Tasmania) (Corporaal, 1950). Questo insetto è stato trasportato in tempi recenti in diversi punti del globo a seguito del massiccio commercio di legname. Molto spesso è riuscito ad acclimatarsi con sorprendente successo nei nuovi territori che ha raggiunto.

Gorham (1876) istituì per questa specie, attribuita dal descrittore al genere Clerus Geoffroy, 1762 (Newman, 1840), Paratillus Gorham, 1876, utilizzato per oltre un secolo, che solo recentemente è stato messo in sinonimia con il genere Tarsostenus Spinola, 1844 (Kolibáć, 2003). Al momento solo 2 specie appartengono a questo genere, precisamente Tarsostenus univittatus (Rossi, 1792) e appunto Tarsostenus carus (Newman, 1840). Entrambe hanno mostrato una spiccata tendenza ad essere diffuse dalle attività antropiche (commercio del legname soprattutto) divenendo in pratica cosmopolite. Risultano essere le uniche rappresentanti della sottofamiglia Tarsosteninae presenti nella regione oloartica.

Secondo la recente classificazione proposta da Opitz (2010), che suddivide la famiglia Cleridae in 12 sottofamiglie, la sottofamiglia Tarsosteninae Jacquelin du Val, 1862 conta complessivamente 19 generi con una settantina di specie, distribuite quasi esclusiva- mente in Australia, Madagascar, Sud Africa e Sud America meridionale (Opitz, 2012).

Materiale esaminato: Marche, Osimo (AN), 28.VI.1999, A. Strocchi leg. (1 9 in coll. I. Zappi) (Fig. 1).

\section{DisCUSSIONE}

Tarsostenus carus risulta essere una specie molto variabile nelle dimensioni, generalmente comprese tra i 4 e i $7 \mathrm{~mm}$, e nella colorazione. Proprio la notevole variabilità cromatica ha portato in passato alla definizione di una serie di "aberrazioni", alcune delle quali riconosciute come tali solo a metà del ' 900 , poiché originariamente descritte o come entità distinte o come varietà geografiche (Corporaal, 1949).

La specie è stata descritta su un esemplare raccolto ad Adelaide (Australia) (Newman, 1840) e successivamente è stata segnalata per l'Australia, la Nuova Caledonia e la Tasmania (Gorham, 1878). In seguito, Kraatz ha descritto la var. sumatranus (Kraatz, 1899) basandosi su un esemplare dell'isola di Sumatra, che per il suo particolare melanismo costituisce una delle aberrazioni meno frequenti. E' nel XX secolo che la specie inizia a comparire in Europa e non solo. Nel 1933 si ha la prima segnalazione per la Gran Bretagna (Donisthorpe, 1933a, 1933b, 1944) e negli anni seguenti la specie viene rinvenuta in diverse lo-

*Iuri Zappi, Via Lido 78, 40033 Casalecchio di Reno (BO), Italia. E-mail: iurizappi@hotmail.com 
calità del Paese, contestualmente si stabilisce il suo status di introduzione permanente (Blair, 1942; Fisher, 1944). Nel 1954, un primo esemplare è raccolto in Sud Africa a Pretoria (Winkler, 1960). Successivamente Tarsostenus carus fa la propria comparsa nel 1983 in Europa continentale e precisamente in Francia a Frayssinet nel dipartimento del Lot (Menier \& Burle, 1985). L'anno seguente vengono segnalati i primi

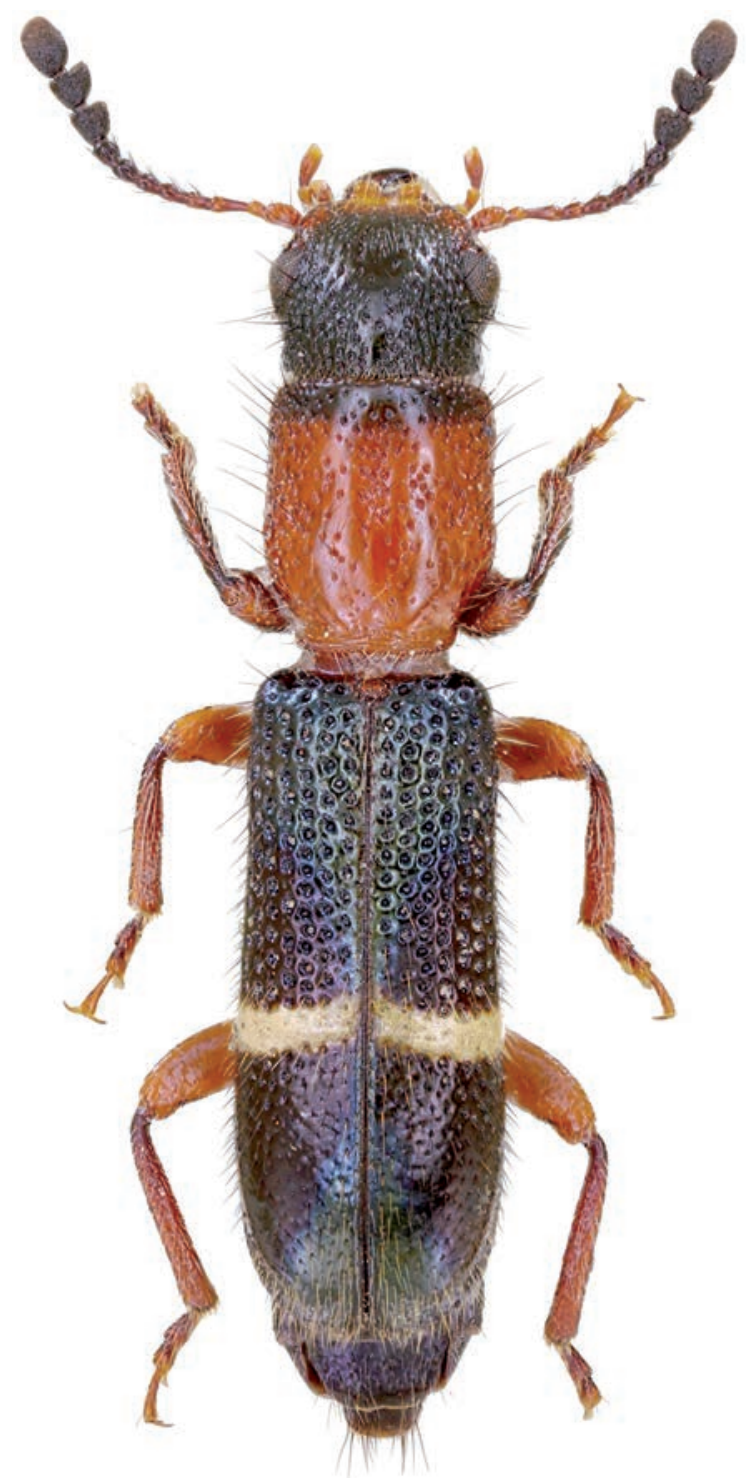

Fig. 1. Tarsostenus carus (Newman, 1840) [O+; Osimo (Ancona)]: habitus. Lunghezza: $6,1 \mathrm{~mm}$. esemplari negli Stati Uniti (Contea di Santa Cruz, California (Grace \& Wood, 1985). Il ritrovamento nelle Marche costituisce la prima segnalazione per l'Italia.

Tarsostenus carus dimostra una elevata capacità adattativa e una notevole velocità di espansione nei nuovi territori colonizzati. Esaminando il caso della Francia, paese che ha visto in questi anni un notevole numero di contributi scientifici volti al monitoraggio di questa specie, si può riscontrare che nel 1990 Tarsostenus carus era noto in soli 2 dipartimenti, nel 2002 in già 13 dipartimenti, mentre nel 2010 la sua presenza è stata accertata in ben 28 dipartimenti (Chapelin-Viscardi, 2009, 2010). Non è pertanto difficile ipotizzare che Tarsostenus carus possa espandersi in un prossimo futuro e con notevole rapidità anche sul territorio italiano. Tra i punti di forza che questa specie ha dimostrato di possedere e che le consentono di acclimatarsi con successo in ambienti molto eterogenei, bisogna ricordare la lunga fenologia (alle latitudini dell'Europa occidentale l'adulto compare in natura da maggio fino ai primi di settembre), la buona attitudine al volo e la grande adattabilità alle diverse essenze arboree. Gli adulti o gli stadi preimaginali sono stati rinvenuti su Eucalyptus sp. (Fisher, 1944), Quercus sp. (Grace \& Wood, 1985) e Ficus carica (Brustel, 2001), ma molte altre essenze vegetali sono considerate sue probabili o potenziali piante ospiti (ad esempio Vitis vinifera e Betula sp.; per un elenco completo si veda Chapelin-Viscardi, 2009). Questo quadro bionomico consente di ipotizzare che Tarsostenus carus sia di fatto caratterizzato da una notevole plasticità ecologica e che possa colonizzare qualunque ambiente sia frequentato dalle proprie prede. Nella sua area d'origine, più precisamente in Australia, la specie è considerata un attivo predatore di coleotteri xilofagi. In Gran Bretagna, Francia e California, Tarsostenus carus risulta essere apparentemente legato soprattutto ai rappresentanti del genere Lyctus (Coleoptera, Bostrichidae, Lyctinae), che preda sia allo stadio larvale che imaginale (Fisher, 1944). In considerazione della sua riconosciuta plasticità ecologica, è quindi estremamente probabile che questo Cleridae sia in grado di rivolgere la propria attività predatoria anche su coleotteri appartenenti alle famiglie Bostrichidae e Curculionidae (Scolytinae) (Chapelin-Viscardi, 2009), così come su altri coleotteri xilofagi o saproxilici.

Per quanto riguarda le modalità di introduzione 
della specie in Italia si potrebbero avanzare diverse ipotesi. Tra le tante possibili, forse le più plausibili potrebbero contemplare l'arrivo della specie via mare mediante un carico di legname scaricato presumibilmente al vicino porto di Ancona, oppure via terra al seguito di una partita di legno forse di provenienza europea e destinata a qualche locale laboratorio di falegnameria.

\section{RINGRAZIAMENTI}

I miei più vivi ringraziamenti vanno ad Alberto Strocchi, che mi ha permesso di studiare l'esemplare da lui raccolto; a Roberto A. Pantaleoni, per l'attenta lettura del manoscritto e i preziosi consigli; agli amministratori e collaboratori del FEI (Forum Entomologi Italiani), per aver creato le premesse per la realizzazione del presente lavoro; ad Augusto Degiovanni, per la bella fotografia.

BIBLIOGRAFIA

Blair K.G., 1942 - The capture of Paratillus carus Newm. (Col. Cleridae) in Sussex. The Entomologist's Monthly Magazine, LXXVIII, 932: 13.

BRUStel H., 2001 - “Oh, cette Grésigne !’. Données faunistiques nouvelles pour cette forêt (Coleoptera), (deuxième supplément au catalogue de Jean Rabil, 1992, 1995). Bulletin de la Société entomologique de France, 106(5): 473-482.

ChaPelin-VisCARDi J-D., 2009 - Sur la chorologie, phénologie et écologie d'un Cléride exotique en France: Paratillus carus (Newman, 1840) (Coleoptera, Cleridae). Bulletin de la Société entomologique de France, 114(3): 365-372.

Chapelin-Viscardi J-D., 2010 - Compléments sur la répartition de Paratillus carus (Newman, 1840) (Col., Cleridae). Bulletin de la Société entomologique de France, 115(2): 165-166.

CorporaAl J.B., 1949 - The variability of Paratillus carus Newm. (Col., Cleridae) $\left(34^{\text {th }}\right.$ communication on Cleridae). The Entomologist's Monthly Magazine, LXXXV(4) X-1021: 156.

CorporaAl J.B., 1950 - Pars 23. Cleridae, pp. 1-373. In: Coleopterorum Catalogus Supplementa. (HINKs W.D., Ed). Uitgeverij Dr. W. Junk, 's-Gravenhage, Netherlands.

Donisthorpe H., 1933a - A beetle new to Britain. Proceedings of the Royal Entomological Society of London, VII: 112. [Tarsostenus carus viene citato come Denops albofasciatus. L'errore di determinazione sarà corretto dallo stesso Donisthorpe nel 1944].

DonisthorPe H., 1933b - Denops albofasciatus Charp. (Cleridae, Col.) captured in Britain. Entomologist's Record, XLV(12): 164. [idem come sopra].

Donisthorpe H., 1944 - Paratillus carus Newm. (Col., Cleridae) probably from Windsor Forest. Berks. - The Entomologist's Monthly Magazine, LXXX: 161.

Fisher R.C., 1944 - A note on Paratillus carus Newm. (Col. Cleridae) and records of its occurrence in Great-Britain. The Entomologist's Monthly Magazine, LXXX - 1009: 132-134.

Gorham H.S., 1878 - Descriptions of new genera and species of Cleridae, with notes on the genera and corrections of synonymy. Transactions of the Entomological Society of London 1878, part. II: 153-167.

Grace J.K., Wood D.L., 1985 - An introduced clerid, Paratillus carus (Newman) (Coleoptera: Cleridae), preying on Lyctus brunneus (Stephens) (Coleoptera: Lyctidae) in California live oak. Pan-Pacific Entomologist, 61(4): 348.

KoliBÁČ J., 2003 - A review of Australian genera of Korynetinae (Coleoptera, Cleridae). Entomologica Basiliensia, 25: 41-97.

Menier J.J., Burle F., 1985 - Première capture en France de Paratillus carus, Cleridae de la Région Australienne (Coleoptera). L'Entomologiste, 41(1): 9-15.

NeWMan E., 1840 - Entomological notes. The Entomologist, I (art.1): 1-16.

OPITZ W., 2010 - Classification, natural history, phylogeny, and subfamily composition of the Cleridae and generic content of the subfamilies (Coleoptera: Cleroidea). Entomologica Basiliensia et Collectionis Frey, 32: 31-128.

OpITz W., 2012 - Classification, natural history, and evolution of Tarsosteninae (Coleoptera: Cleridae) - Part I: Generic composition of the subfamily and key and phylogeny of genera. Psyche, 2012: 1-35.

WinkLeR J.R., 1960 - Chapter VIII. Coleoptera: Cleridae. In: South African Animal Life, Vol. VII: 128-162. 